\title{
EQUIVALENCE RELATIONS IN ALGEBRAIC GEOMETRY
}

\section{ERNST SNAPPER}

1. The cycle groups $C_{s}$. An algebraic variety $V$ in $n$-dimensional complex projective space $P^{(n)}$ is obtained by equating to zero a finite number of forms $F_{1}\left(x_{0}, \cdots, x_{n}\right), \cdots, F_{m}\left(x_{0}, \cdots, x_{n}\right)$ with complex coefficients; $V$ is assumed to be nonempty. If $V$ is irreducible, that is, if $V$ is not the union of a finite number of proper subvarieties, it is possible to associate with $V$ in several ways a complex dimension $d$. For example, just as $P^{(1)}$ is topologically equivalent to a real 2dimensional sphere, so can every $P^{(n)}$ be represented topologically by a $2 n$-dimensional real complex in the sense of combinatorial topology. (See [1]; numbers in brackets refer to the references.) In this representation, $V$ goes over into an even-dimensional, connected, orientable, closed complex whose dimension is defined as $2 d$. This complex is denoted by $K^{(2 d)}$ and $V$ itself by $V^{(d)}$.

Consider the set $T_{s}$ of irreducible, $s$-dimensional subvarieties of $V^{(d)}$ for some fixed $s$, where $0 \leqq s \leqq d$. A function on $T_{s}$ is called integral if its value for every element of $T_{s}$ is a rational integer, and if the function is zero except for at most a finite number of elements of $T_{s}$; these functions constitute of course an additive group, denoted by $C_{s}$. We identify the integral function which at the elements $W_{1}^{(s)}, \cdots, W_{h}^{(s)}$ of $T_{s}$ assumes the values $n_{1}, \cdots, n_{h}$ and which is zero everywhere else on $T_{s}$ with the linear combination $n_{1} W_{1}^{(s)}+\cdots$ $+n_{h} W_{h}^{(s)}$. Since every $W_{i}^{(s)}$ gives rise to a $2 s$-dimensional, connected, closed, orientable subcomplex of $K^{(2 d)}$, the above linear combination can be interpreted as a $2 s$-dimensional cycle of $K^{(2 d)}$ in the sense of topology. This fact is the reason why we call the elements of $C_{s}$ the $s$-dimensional cycles of $V^{(d)}$ and often consider $C_{s}$ as a subgroup of the $2 s$-dimensional cycle group of $K^{(2 d)}$. A cycle is called effective if, considered as a function, it never assumes a negative value; otherwise the cycle is called virtual. The effective cycles are clearly closed under addition but not under subtraction, and every cycle is the difference of two effective cycles.

The group $C_{s}$ is completely determined by the cardinal number of $T_{s}$, and hence its structure is of no interest. The importance of $C_{s}$ lies in the fact that the different aspects of the geometry of $V^{(d)}$ are most conveniently studied by means of the equivalence relations which

An address delivered before the Palo Alto meeting of the Society on May 2, 1953 by invitation of the Committee to Select Hour Speakers for Far Western Sectional Meetings; received by the editors May 18, 1953. 
they impose upon $C_{8}$. Let us first study equivalence relations abstractly.

2. Abstract equivalence relations. Let $G$ be an additive group, and let $E$ be a nonempty subset of $G$ which is closed under addition but not necessarily under subtraction. We furthermore assume that each element of $G$ is the difference of two elements of $E$. In our applications, $G$ will be $C_{s}$ and $E$ the set of effective cycles of $C_{s}$.

Suppose that a binary relation $\approx$ has been defined for just the elements of $E$ which is reflexive, symmetric, and additive. Additivity means of course that if four elements $E_{1}, E_{2}, E_{3}, E_{4}$ of $E$ are such that $E_{1} \approx E_{2}$ and $E_{3} \approx E_{4}$, then $E_{1}+E_{3} \approx E_{2}+E_{4}$. In terms of $\approx$ we define a binary relation $*$ for all of $G$ by postulating that for two elements $A$ and $B$ of $G, A * B$ when there exists an $X \in G$ such that $A+X$ and $B+X$ belong to $E$ and $A+X \approx B+X$. (It is clear that $X$ can always be chosen in $E$.)

Statement 2.1. The binary relation * for all of $G$ is reflexive, symmetric, transitive, and additive.

Proof. If $A \in G$, then $A=E_{1}-E_{2}$ where $E_{1}, E_{2} \in E ; A * A$ follows from using $E_{2}$ as $X$. The symmetry and additivity of $*$ follow immediately from the corresponding properties of $\approx$. If $A * B$ and $B * C$, the additivity of $*$ implies that $A+B * B+C$. Hence there exists an $X^{1} \in G$ such that $A+B+X^{1}$ and $B+C+X^{1}$ belong to $E$ and $A+B+X^{1} \approx B+C+X^{1}$. Using then $B+X^{1}$ as $X$, we conclude that $A * C$.

According to (2.1), the relation * divides the elements of $G$ into mutually exclusive classes, which can be considered as the elements of a homomorphic image of $G$ with respect to the subgroup $F$ which consists of all elements $A * O$ as kernel. ( $O$ is the zero element of $G$.) Since $A * O$ means that there exists an $X \in E$ such that $A+X \approx X$, we can also say that $F$ consists of all elements of $G$ which can be written as $E_{1}-E_{2}$, where $E_{1}, E_{2} \in E$ and $E_{1} \approx E_{2}$.

It is clear that if $E_{1}, E_{2} \in E$ and $E_{1} \approx E_{2}$, then $E_{1} * E_{2}$ while the converse may not hold. (Algebraic equivalence gives an example of this.) However, if $\approx$ is also subtractive on $E$, we prove immediately that $\approx$ and $*$ coincide on $E$. ( $\approx$ is of course called subtractive if $E_{1}, E_{2}, E_{3}, E_{4} \in E$ and $E_{1} \approx E_{2}, E_{3} \approx E_{4}$, and $E_{1}-E_{3} \in E$ and $E_{2}-E_{4}$ $\in E$ imply that $E_{1}-E_{3} \approx E_{2}-E_{4}$.)

In algebraic geometry it may happen that a binary relation $\sim$ is defined for the elements of $E$ which is only reflexive and symmetric but not additive. (Rational equivalence gives an example of this.) We then first go over to a new binary relation $\approx$ for $E$ by defining that, for two elements $E_{1}$ and $E_{2}$ of $E, E_{1} \approx E_{2}$ if there exist elements 
$E_{11}, \cdots, E_{1 h} \in E$ and $E_{21}, \cdots, E_{2 h} \in E$ such that $E_{1}=E_{11}+E_{12}$ $+\cdots+E_{1 h}, E_{2}=E_{21}+E_{22}+\cdots+E_{2 h}$, and $E_{1 i} \sim E_{2 i}$ for $i=1, \cdots$, $h$; clearly $\approx$ is reflexive, symmetric, and additive on $E$. The relation $\sim$ is usually given by means of a covering $U$ of $E$, where $E_{1} \sim E_{2}$ then means that $E_{1}$ and $E_{2}$ belong to at least one element of $U$. The above additive relation $\approx$ is then determined by the covering of $E$ which consists of all elements of $U$ together with those subsets of $E$ which are sums of a finite number of elements of $U$. (If $U_{1}, \cdots, U_{h}$ $\in U, U_{1}+\cdots+U_{h}$ is the subset of $E$ which consists of all sums $E_{1}+\cdots+E_{h}$ where $E_{i} \in U_{i}$ for $i=1, \cdots, h$.)

Every covering $U$ of any set whatsoever is a partially ordered system, obtained by ordering the elements of $U$ by inclusion. We call $U$ an inductive covering if the partially ordered system $U$ is inductive, that is, if every totally ordered subsystem of $U$ has an upper bound in $U$. According to Zorn's lemma, every element of $U$ is then contained in a maximal element of $U$, and hence the maximal elements of $U$ then constitute a new covering $M$ of the set which clearly defines the same binary relation $\sim$ as $U$. The following remarks concerning $U$ and $M$ are useful, as all coverings which occur in algebraic geometry are inductive.

STATEMENT 2.2. The elements of $M$ are mutually exclusive subsets if and only if any two elements of $U$ which have a nonempty intersection are both contained in the same element of $U$. Clearly, the relation $\sim$ is then transitive.

Proof. Let the elements of $M$ be mutually exclusive and let $U_{1}, U_{2} \in U$ where $U_{1} \cap U_{2} \neq \varnothing . U_{1}$ and $U_{2}$ are contained in respectively the elements $M_{1}$ and $M_{2}$ of $M$, and since clearly $M_{1}=M_{2}$, the desired result follows. Conversely, if the condition on $U$ is satisfied and $M_{1}, M_{2} \in M$, where $M_{1} \neq M_{2}$, then $M_{1} \cap M_{2}$ must be empty since distinct maximal elements of $U$ can not be contained in the same element of $U$.

In algebraic geometry, the elements of $U$ are always certain irreducible algebraic systems of effective cycles. Before discussing algebraic systems, let us indicate by means of an example how the above notions may be used in topology.

EXAMPLE 2.1. Let $K$ be a complex in the sense of topology, and let $G$ be the additive group of the $s$-dimensional cycles of $K$. Hence $G$ consists of the finite, linear combinations with integral coefficients of the connected, closed, oriented $s$-dimensional subcomplexes of $K$. Choosing $E=G$, we say that $A \sim B$, where $A, B \in G$, if both $A$ and $B$ can be deformed continuously into the same $s$-dimensional cycle. It is trivial that $\sim$ is reflexive and symmetric and hence * defines a 
homomorphic image $J$ of $G$ which is obviously a topological invariant of $K$. Clearly, if $A * B$, these two cycles are homologous in the sense of topology and consequently the $s$-dimensional homology group of $K$ is a homomorghic image of $J$. Whenever for a complex the topological relation of homology coincides with the relation $*$, we are in the advantageous position of being able to define homology solely by notions which refer to continuous deformations. In this connection, it is interesting to observe that the well known one-dimensional cycle $A$ of the double torus, which is homologous to zero but can not be deformed continuously into a point, nevertheless satisfies $A * O$. ( $O$ is the zero-cycle.)

3. Algebraic systems. Returning to $\S 1$, let $A=n_{1} W_{1}^{(s)}+\cdots$ $+n_{h} W_{h}^{(s)}$ be a $d$-dimensional cycle of an irreducible algebraic variety $V^{(d)}$. We denote by $\partial_{i}$ the degree of the irreducible variety $W_{i}^{(s)}$, that is, the maximum number of points of intersection which can occur when $W_{i}^{(s)}$ is intersected with a linear, $(n-s)$-dimensional subspace $L^{(n-s)}$ of the projective space $P^{(n)}$, where neither $W_{i}^{(s)} \subset L^{(n-s)}$ nor $L^{(n-s)} \subset W_{i}^{(s)}$. The rational integer $\partial=n_{1} \partial_{1}+\cdots$ $+n_{h} \partial_{h}$ is called the degree of $A$. It was proved in [2] that, for fixed $s$ and $\partial$, there exists an algebraic correspondence $N$ from an algebraic variety $\Pi$ onto $V^{(d)}$ which establishes a (1-1)-correspondence between the points of $\Pi$ and all the effective cycles of dimension $s$ and degree $\partial$ of $V^{(d)} ; \Pi$ and $N$ depend of course on $s$ and $\partial$. Precisely, $\Pi$ is a subvariety of some projective space $P^{(t)}$ described by coordinates $y_{0}, \cdots, y_{t}$; that is, the forms which determine $\Pi$ are forms in the variables $y_{0}, \cdots, y_{t} ; t$ depends also on the choice of $s$ and $\partial$ and $\Pi$ may be reducible. $N$ is determined by a finite set of forms $k_{1}(x ; y), \cdots, k_{a}(x ; y)$ (with complex coefficients) which are homogeneous separately in $x_{0}, \cdots, x_{n}$ and $y_{0}, \cdots, y_{t}$. These forms are such that if $\left(\eta_{0}, \cdots, \eta_{t}\right)$ is a point of $\Pi$ and $N\left(\eta_{0}, \cdots, \eta_{t}\right)$ $=n_{1} W_{1}^{(s)}+\cdots+n_{h} W_{h}^{(s)}$, the algebraic variety $W_{1}^{(s)} \cup \cdots \cup W_{h}^{(s)}$ is determined by the equations $k_{1}(x ; \eta)=0, \cdots, k_{a}(x ; \eta)=0$. The positive multiplicities $n_{1}, \cdots, n_{h}$ can be defined conveniently by means of the "associated forms" of [2] and hence their definition does not require any theory of "specialization."

The collection of effective cycles which corresponds under $N$ to an algebraic subvariety $\Omega$ of $\Pi$ is called an algebraic system. Dimension, irreducibility, and components of an algebraic system $S$ are defined in terms of its "parameter variety $\Omega . "$

In general, an algebraic correspondence $J$ from a variety $V_{0}$ onto $V^{(d)}$ is a correspondence defined by means of a finite set of doubly homogeneous forms, just as $N$ was defined by the forms $k_{i}(x ; y)$. 
The points of $V_{0}$ are transformed by $J$ into effective cycles of $V^{(d)}$; and, if all these cycles have the same dimension and degree, they constitute an algebraic system. This is usually the easiest way to check whether a given collection of cycles is an algebraic system. If $V_{0}$ is also irreducible, $J$ is called a birational transformation if the correspondence is a (1-1)-point transformation except at most on proper subvarieties of $V_{0}$ and $V^{(d)}$. We say then that $V_{0}$ and $V^{(d)}$ are birational models of one another. A large part of algebraic geometry consists of searching for birational invariants, that is, invariants of the variety under consideration which do not change when the variety is submitted to birational transformations. Dimension is a birational invariant. If $\Omega$ is the parameter variety of an algebraic system $S$ and $J$ is an algebraic correspondence from some other algebraic variety $\Omega^{*}$ onto $\Omega$ which is a (1-1)-point transformation without exception, we can clearly also consider $\Omega^{*}$ as a parameter variety of $S$ with $N$ replaced by $N J$.

Examples 3.1. The points of $V^{(d)}$, that is, $s=0, \partial=1$, constitute an irreducible algebraic system of dimension $d$ with $V^{(d)}$ as parameter variety. The point groups of arbitrary degree $\partial$ (those for which $s=0$ and $\partial$ is arbitrary) constitute a $(\partial d)$-dimensional, irreducible algebraic system with the product $V^{(d)} \times \cdots \times V^{(d)}$ of $V^{(d)}$ with itself $\partial$ times as parameter variety. The straight lines on an irreducible quadratic surface $V^{(2)}$ in 3 -space (here $s=\partial=1$ ) constitute a reducible algebraic system with two irreducible 1-dimensional components, namely the two pencils of lines on $V^{(2)}$; observe that these pencils have no element in common. Choose $V^{(3)}=P^{(3)}$, that is, all of 3 -space. When $s=\partial=1$, we obtain again the straight lines, and it is well known that they constitute an irreducible 4-dimensional algebraic system whose parameter variety $\Omega^{(4)}$ arises from the quadratic relations among the Plücker coordinates. Let us consider the 2nd degree curves of $P^{(3)}$; then $s=1, \partial=2$. We have in the first place the pairs of lines, and they constitute an irreducible 8-dimensional system with $\Omega^{(4)} \times \Omega^{(4)}$ as parameter variety. Secondly, we have all conics. The conics which lie in a fixed plane of $P^{(3)}$ form an irreducible 5-dimensional system and hence the conics of $P^{(3)}$ constitute an 8-dimensional, irreducible system. Consequently for $s=1, \partial=2$, we obtain a reducible algebraic system with two irreducible 8-dimensional components; observe that these components have a nonempty intersection, namely the 7-dimensional algebraic system consisting of the pairs of intersecting lines. It may happen that $\Pi$ consists of only a finite number of points, even when $s<d$. For example, when $V^{(2)}$ is a nonsingular cubic surface in $P^{(3)}, \Pi$ for $s=\partial=1$ consists of 27 points. 
Warning. Let $\Omega$ be the irreducible parameter variety of an irreducible algebraic system $S$. If $A_{1}, A_{2} \in S$ and $Q_{1}, Q_{2}$ are the points of $\Omega$ which correspond respectively to $A_{1}$ and $A_{2}$, we can join $Q_{1}$ and $Q_{2}$ by an arc which lies completely on $\Omega$. We should not conclude from this that necessarily $A_{1}$ can be continuously deformed into $A_{2}$. For example, if $S$ is the irreducible 8-dimensional system consisting of all pairs of lines of $P^{(3)}$ and $A_{1}$ is a pair of intersecting lines and $A_{2}$ is a pair of not intersecting lines, $A_{1}$ can not be deformed continuously into $A_{2}$; of course, in this case it is still possible to deform $A_{2}$ continuously into $A_{1}$.

Finally, we observe that irreducible algebraic systems satisfy both the ascending and the descending chain conditions. This result follows from the fact that the irreducible systems, belonging to either an increasing or a descending chain, can all be considered as irreducible subvarieties of the fixed variety $\Pi$ which comprises all effective cycles of the dimension and the degree under consideration.

4. Algebraic equivalence. Consider the cycle group $C_{s}$ for some fixed dimension $0 \leqq s \leqq d$ of our $d$-dimensional irreducible algebraic variety $V^{(d)}$. For two effective cycles $E_{1}, E_{2} \in C_{s}$ we define that $E_{1} \approx E_{2}$ if they belong to the same irreducible algebraic system; $E_{1}$ and $E_{2}$ then have the same degree. The relation $\approx$ is trivially reflexive and symmetric, and it is easily seen to be additive. For, if $E_{1}$ and $E_{2}$ belong to an irreducible algebraic system with parameter variety $\Omega_{1}$, and if $E_{3}$ and $E_{4}$ belong to an irreducible algebraic system with parameter variety $\Omega_{2}$, then $E_{1}+E_{3}$ and $E_{2}+E_{4}$ belong to the necessarily irreducible algebraic system whose parameter variety is the product $\Omega_{1} \times \Omega_{2}$. We conclude from $\S 2$ that we obtain a homomorphic image of $C_{s}$ by defining that $A\|\| B$ if $A$ and $B$ are any two elements of $C_{s}$ for which there exists an $X \in C_{s}$ such that $A+X$ and $B+X$ are effective and $A+X \approx B+X$; clearly $A$ and $B$ must then have the same degree. The equivalence relation |||| is called algebraic equivalence. The relation $\approx$ was obtained from the covering $U$ of the set of effective cycles of $C_{s}$ whose elements are the irreducible algebraic systems. Since irreducible algebraic systems satisfy the ascending chain condition, $U$ is inductive; $M$ (see $\$ 2$ ) consists of the irreducible components of the different algebraic systems which comprise all effective $s$-dimensional cycles of fixed degree $\partial$. (See $\S 3$. We have one such system for each $\partial=1,2, \ldots$.) The sets of $M$ may not be mutually exclusive, as the 2 nd degree curves of $P^{(3)}$ demonstrate (see examples 3.1), and consequently $\approx$ and |||| may not coincide for the effective cycles of $C_{8}$. For example, choosing $V^{(d)}=P^{(3)}$, a nonsingular conic $A$ and a pair of not intersecting lines $B$ are in the rela- 
tion |||| but not in the relation $\approx$. (Again, $A$ can not be deformed continuously into $B$, but now $A$ and $B$ can both be deformed continuously into the same pair of intersecting lines. Considerations of this sort gave rise to example 2.1.) In spite of the warning of $\$ 3$, when studying algebraic equivalence in $C_{s}$, we can expect to be dealing with questions closely tied up with the homology relation of the $2 s$-dimensional cycles of $K^{(2 d)}$. When $d=2$, this is exactly the case as we will now explain.

Let $V^{(2)}$ be an irreducible algebraic surface in 3-dimensional space whose complex $K^{(4)}$ can be considered as homogeneous; this means that every point of $K^{(4)}$ is the interior of a 4-cell. Every surface has a birational model of this type. This follows from the classical result that every surface has a birational model without singularities in 5-dimensional projective space and hence one with at most so-called "ordinary singularities" in 3-dimensional space. For our $K^{(4)}$, the Poincaré duality relations are now valid and hence, if $b_{0}, b_{1}, b_{2}, b_{3}, b_{4}$ are its Betti numbers and $\sigma_{0}, \sigma_{1}, \sigma_{2}, \sigma_{3}$ its torsion coefficients, $b_{0}=b_{4}$ $=1, b_{1}=b_{3}, \sigma_{0}=\sigma_{3}=0, \sigma_{1}=\sigma_{2}$. In order to investigate $b_{2}$ and $\sigma_{2}$, we study $C_{1}$ under algebraic equivalence. It was proved by Lefschetz (see [3]) that if $A, B \in C_{1}$, then $A\|\| B$ if and only if their 2-dimensional images in $K^{(4)}$ are homologous. Hence the homomorphic image $H_{1}$ of $C_{1}$ which |||| defines is isomorphic to a subgroup of the 2-dimensional homology group of $K^{(4)}$. Since this homology group has a finite number of generators with respect to the integers, so does $H_{1}$; and consequently $H_{1}$ is a direct sum $H_{1}=T \oplus B$, where $T$ consists of all the elements of $H_{1}$ which have a nonzero order. We should expect that $T$ is isomorphic with a subgroup of the 2-dimensional torsion group of $K^{(4)}$ but, as is proved in [3], $T$ actually is isomorphic with that torsion group itself. Hence, the minimum number of generators of $T$ is the algebro-geometric definition of $\sigma_{1}=\sigma_{2} . B$ is indeed isomorphic with only a subgroup of the 2-dimensional Betti group of $K^{(4)}$ and hence, if $\rho$ denotes the maximum number of linearly independent elements of $B, \rho \leqq b_{2}$. A birational transformation which either produces or loses exceptional curves is not a topological transformation, and hence topological invariants may not be birational invariants. Indeed, neither $\rho$ nor $b_{2}$ are birational invariants, but both numbers change by the amount $e_{1}-e_{2}$ if the transformation produces $e_{1}$ exceptional curves and loses $e_{2}$ such curves. It follows that $b_{2}-\rho=\rho_{0}$ is a birational invariant of $V^{(2)}$. In the transcendental theory of algebraic surfaces, $\rho$ occurs in the study of the logarithmic singularities of the simple integrals of the third kind of $V^{(2)}$, while the birational invariance of $\rho_{0}$ also follows from the fact that $V^{(2)}$ 
possesses exactly $\rho_{0}$ proper double integrals of the second kind which are linearly independent modulo the improper integrals. Every birational transformation can be decomposed into a finite number of birational transformations, each one of which produces or loses only exceptional curves of the first kind. Since such curves can not belong to $T$ (the "virtual degree" of an exceptional curve of the first kind is -1 and of a curve of $T$ is 0$), \sigma_{2}=\sigma_{1}$ is also a birational invariant of $V^{(2)}$.

EXAMPLES 4.1. If $V^{(2)}$ is a plane, all effective, one-dimensional cycles of degree $\partial$ form an irreducible algebraic system of dimension $\partial(\partial+3) / 2$, parametrized by the projective space whose coordinates are the coefficients of the forms $k\left(x_{0}, x_{1}, x_{2}\right)$ of degree $\partial$. Hence if $A$ is a straight line of $V^{(2)}$ all these cycles are algebraically equivalent to $\partial A$. Since every cycle is the difference of two effective cycles, every cycle is algebraically equivalent to $n A$ for some whole number $n$. It follows that $\rho=1$ and that $\sigma_{1}=\sigma_{2}=0$. A surface is called rational if it can be transformed birationally into a plane. Hence, the torsion coefficients of rational surfaces are always zero. Let $V^{(2)}$ be a nonsingular quadric. If we project $V^{(2)}$ on a plane from a point $Q$ on $V^{(2)}$ as projection center, in the resulting birational transformation the two straight lines of $V^{(2)}$ which pass through $Q$ go over into points and $Q$ itself goes over in a straight line. Hence, $V^{(2)}$ is a rational surface, and $e_{1}=1, e_{2}=2$; consequently $\rho+1-2=1$, that is, $\rho=2$. Two straight lines $A, B$ of $V^{(2)}$ which intersect are not algebraically equivalent and hence every one-dimensional cycle of $V^{(2)}$ is algebraically equivalent to $n_{1} A+n_{2} B$. Surfaces with nonzero torsion are the sixth degree surfaces whose ordinary singularities form the six edges of a tetrahedron. These surfaces, which consequently are never rational, are due to Enriques.

It is believed that not only the $C_{1}$ of $V^{(2)}$ has a finite number of generators with respect to \|\| , but that this is true for every $C_{s}$ of every $V^{(d)}$ with respect to any groundfield of characteristic zero. This conjecture has been proved for $s=d-1$ and the complex numbers as groundfield by means of transcendental and topological methods. Beyond this case, the conjecture has been proved only for special types of varieties. Even when $d=2$, we can not prove the conjecture when the groundfield is not the field of complex numbers. ${ }^{1}$

5. Linear series. In the theory of linear equivalence, arbitrary ir-

${ }^{1}$ A proof for the case $s=d-1$ and arbitrary groundfield is sketched by A. Néron in La théorie de la base pour les diviseurs sur les variêtés algêbriques, "Deuxième Colloque de Géométrie Algébrique," Liège, 1952, pp. 119-126. 
reducible algebraic systems are replaced by the special irreducible algebraic systems which are called linear series. So, let us study them first.

Consider a form $k\left(x_{0}, \cdots, x_{n}\right)$ of positive degree which is such that the hypersurface $G$ defined by $k(x)=0$ does not contain our $V^{(d)}$. The intersection $V^{(d)} \cap G$ is then the union of a finite number of irreducible, $(d-1)$-dimensional subvarieties of $V^{(d)}$, say $V^{(d)} \cap G$ $=W_{1}^{(d-1)} \cup \ldots \cup W_{h}^{(d-1)}$. It is proved in [4] that the intuitive intersection multiplicities which a geometer wants to associate with the intersection components $W_{1}^{(d-1)}, \ldots, W_{h}^{(d-1)}$ can be defined idealtheoretically as follows. Let $\mathfrak{p}$ be the prime ideal which consists of all forms which vanish everywhere on $V^{(d)}$. In a decomposition of the ideal $(\mathfrak{p}, k(x))$ into largest primary ideals, exactly $h$ isolated primary components occur, say $\mathfrak{q}_{1}, \cdots, \mathfrak{q}_{h}$. The associated prime ideal $\mathfrak{p}_{i}$ of $\mathfrak{q}_{i}$ defines a variety $W_{i}^{(d-1)}$, and the intersection multiplicity $n_{i}$ of $W_{i}^{(d-1)}$ as a component of $V^{(d)} \cap G$ is the length of the primary ideal $\mathfrak{q}_{i}, i=1, \cdots, h$. It is well known that lengths of primary ideals do not generally give the correct multiplicities in intersection theory, but in our simple case, where a variety is intersected with a hypersurface, the length can always be used. From now on we consider $V^{(d)} \cap G$ as the effective $(d-1)$-dimensional cycle $n_{1} W_{1}^{(d-1)}+\ldots$ $+n_{h} W_{h}^{(d-1)}$. If the degrees of $V^{(d)}$ and $G$ are respectively $\alpha$ and $\beta$ (it is easy to show that $\beta$ is the degree of the form $k\left(x_{0}, \cdots, x_{n}\right)$ ), the degree of the cycle $V^{(d)} \cap G$ is $\alpha \beta$. For this reason, when $k\left(x_{0}, \cdots, x_{n}\right)$ is a nonzero constant and hence $G$ the empty set, we consider $V^{(d)} \cap G$ as the zero cycle.

EXAMPLE 5.1. Let $V^{(2)}$ be an irreducible quadratic cone in 3-space and $A$ and $B$ two distinct straight lines on $V^{(2)}$. The plane containing $A$ and $B$ is denoted by $G_{1}$, and the tangent plane of the cone which contains $A$ by $G_{2}$. Then $V^{(2)} \cap G_{1}=1 A+1 B$ and $V^{(2)} \cap G_{2}=2 A$; the multiplicity 2 arises from the fact that if we deform $G_{2}$ into another plane through the vertex of the cone, $V^{(2)} \cap G_{2}$ splits up into two distinct straight lines.

Now let $k_{0}\left(x_{0}, \cdots, x_{n}\right), \cdots, k_{r}\left(x_{0}, \cdots, x_{n}\right)$ be $r$ forms which all have the same degree $\beta$ and which are linearly independent modulo $V^{(d)}$. If $\lambda_{0}, \cdots, \lambda_{r}$ are complex numbers, not all zero, $\lambda_{0} k_{0}(x)+\cdots+\lambda_{r} k_{r}(x)$ is also a form of degree $\beta$ and the condition of linear independence states exactly that the hypersurface $G\left(\lambda_{0}, \cdots, \lambda_{r}\right)$ with equation $\lambda_{0} k_{0}(x)+\cdots+\lambda_{r} k_{r}(x)=0$ does not contain $V^{(d)}$. Hence, the intersection cycle $V^{(d)} \cap G\left(\lambda_{0}, \cdots, \lambda_{r}\right)$ is a well defined effective cycle of degree $\alpha \beta$ of $C_{d-1}$ ( $\alpha$ is again the degree of $\left.V^{(d)}\right)$. The effective cycles, which in this way correspond to the 
points of the $r$-dimensional projective space $\Lambda^{(r)}$ of $(r+1)$-tuples $\left(\lambda_{0}, \cdots, \lambda_{r}\right)$, are called a linear series $g^{r}$ of dimension $r$ and degree $\alpha \beta$. If it is possible to subtract from all the cycles of $g_{\alpha \beta}^{r}$ a fixed effective $(d-1)$-dimensional cycle of degree $\beta_{0}$ so that all the remaining cycles are still effective, we consider also these remaining cycles as a linear series $g_{\alpha \beta-\beta_{0}}^{r}$ of dimension $r$ and degree $\alpha \beta-\beta_{0}$, parametrized by the same projective space $\Lambda^{(r)}$ as above.

It is clear that an $r$-dimensional linear series $g_{t}^{r}$ is an irreducible, $r$-dimensional algebraic system consisting of $(d-1)$-dimensional effective cycles of degree $t$ for whose parameter variety we can choose the above projective space $\Lambda^{(r)}$. It follows that $r$ can not exceed the dimension of the algebraic variety II which parametrizes all the effective $(d-1)$-dimensional cycles of degree $t$. For example, if $d=1, g_{t}^{r}$ consists of pointgroups of degree $t$, and we have seen in examples 3.1 that $\Pi$ then has dimension $t$. Hence, $r \leqq t$, if $g_{t}^{r}$ is a linear series on a curve.

EXAMPLE 5.2. Let $V^{(2)}$ again be an irreducible quadratic cone in 3-space $P^{(3)}$ and $A$ and $B$ two distinct lines on $V^{(2)}$. All quadrics of $P^{(3)}$ which contain both $A$ and $B$ constitute a linear system $L$ of dimension 4 in which occur three quadrics which are linearly independent modulo our $V^{(2)}$. Hence the quadrics of $L$ cut out on the cone a linear series $g_{4}^{3}$, consisting of $A+B+D_{i}$, where $D_{i}$ runs through all the nonsingular and singular conics on the cone. This $g_{4}^{3}$ has $A+B$ as fixed cycle. Consequently the sets of effective cycles $A+D_{i}, B+D_{i}, D_{i}$ are all considered as 3-dimensional linear series of degrees respectively $3,3,2$; only the last linear series has no fixed cycle.

Let $p\left(x_{0}, \cdots, x_{n}\right), q\left(x_{0}, \cdots, x_{n}\right)$ be two forms of the same degree, where the hypersurface $G(q)$ with equation $q(x)=0$ does not contain $V^{(d)}$. The quotient $p(x) / q(x)$ then determines a function on $V^{(d)}$ which is well defined there except for the points of the cycle $V^{(d)} \cap G(q)$. If we add and multiply these functions as functions on $V^{(d)}$, we obtain a field $F$ which is called the field of rational functions of $V^{(d)}$. If also the hypersurface $G(p)$ with equation $p(x)=0$ does not contain $V^{(d)}$, the function is a nonzero element of $F$ and the well defined $(d-1)$-dimensional cycle $\left(V^{(d)} \cap G(p)\right)-\left(V^{(d)} \cap G(q)\right)$ of degree zero is then called the cycle $D(\phi)$ of the rational function $\phi$ determined by $p(x) / q(x)$. It is easy to see that the mapping $\phi \rightarrow D(\phi)$ is a homomorphism from the multiplicative group of $F$ into $C_{d-1}$, and hence these cycles $D(\phi)$ form a subgroup $R$ of $C_{d-1}$. Two irreducible varieties can be mapped onto one another by means of a birational transformation if and only if their fields of rational functions are 
isomorphic under an isomorphism which leaves the constant functions pointwise fixed. Small wonder then that if we study $C_{d-1}$ under the equivalence relation imposed upon it by $R$, and this relation will turn out to be linear equivalence, we shall discover further birational invariants.

Let $E_{1}$ and $E_{2}$ be two effective cycles which belong to the same linear series, cut out on $V^{(d)}$ by the linearly independent forms $k_{0}(x), \cdots, k_{r}(x)$; say $E_{1}$ and $E_{2}$ arise respectively from the hypersurfaces $G_{1}, G_{2}$ with equations $\lambda_{0} k_{0}(x)+\cdots+\lambda_{r} k_{r}(x)=0$ and $\mu_{0} k_{0}(x)$ $+\cdots+\mu_{r} k_{r}(x)=0$. Denoting by $E_{0}$ the fixed divisor which may have been subtracted from the total intersections, $E_{1}=\left(V^{(d)} \cap G_{1}\right)$ $-E_{0}$ and $E_{2}=\left(V^{(d)} \cap G_{2}\right)-E_{0}$. Hence, $E_{1}-E_{2}=D(\phi)$, where $\phi$ is the nonzero rational function of $V^{(d)}$ determined by the quotient $\lambda_{0} k_{0}(x)$ $+\cdots+\lambda_{r} k_{r}(x) / \mu_{0} k_{0}(x)+\cdots+\mu_{r} k_{r}(x)$. We prove just as easily that all cycles of $R$ can be obtained in this way; that is, a cycle belongs to $R$ if and only if it is the difference of two effective cycles which belong to the same linear series. This statement establishes the connection between linear series and rational functions.

6. Linear equivalence. This type of equivalence is restricted to the group $C_{d-1}$ of our $V^{(d)}$. For two effective cycles $E_{1}, E_{2} \in C_{d-1}$ we define that $E_{1} \approx E_{2}$ if they belong to the same linear series, that is, if $E_{1}-E_{2}$ belongs to the subgroup $R$ of $C_{d-1}$. (See the previous section.) We conclude immediately that the relation $\approx$ is reflexive, symmetric, additive, subtractive, and transitive. It follows from $\$ 2$ that we obtain a homomorphic image of $C_{d-1}$ with kernel $R$ by defining that $A \equiv B$ if $A$ and $B$ are any two elements of $C_{d-1}$ for which there exists an $X \in C_{d-1}$ such that $A+X$ and $B+X$ are effective and $A+X \approx B+X$. Clearly, if $A \equiv B$, these cycles have the same degree and the relations $\equiv$ and $\approx$ coincide on the set of effective cycles. Observe that if $A \equiv B$, then certainly $A|||| B$.

The relation $\approx$ was obtained from the covering $U$ of the set of effective cycles of $C_{d-1}$ whose elements are the linear series of $V^{(d)}$. Since linear series are irreducible algebraic systems, $U$ is again inductive. The elements of $M$ (see $\$ 2$ ) are now called complete linear series. In order to show that two distinct complete linear series are mutually exclusive, we must prove that when two linear series have a cycle in common, they are contained in the same linear series. (See statement 2.2.) This fact, whose analogue we have seen to be false for algebraic equivalence, does not follow trivially from anything which has been said in this exposition; a simple proof of it can be found in $\$ 17$ of [5]. Accepting this fact as established, we see that every effective, $(d-1)$-dimensional cycle $E$ is contained in a unique complete linear 
series $|E|$ which consists of all effective cycles, linearly equivalent to $E$; exactly the same remark can be made concerning any linear series $g^{r}$ and the unique complete linear series $\left|g^{r}\right|$ to which all cycles of $g^{r}$ belong. If $\left|g^{r}\right|$ has dimension $r^{1}$, the deficiency $\delta$ of $g^{r}$ is defined as $\delta=r^{1}-r$; clearly $\delta \geqq 0$, and $\delta=0$ if and only if $g^{r}$ is complete.

Let $W^{(d-1)}$ be an irreducible, $(d-1)$-dimensional subvariety of $V^{(d)}$ and let us choose $E=W^{(d-1)}$. Suppose that $|E|$ has dimension $r$ and is cut out on $V^{(d)}$ by the hypersurfaces of the system $\lambda_{0} k_{0}(x)$ $+\cdots+\lambda_{r} k_{r}(x)=0$, where of course an appropriate fixed, effective cycle $E_{0}$ may have been subtracted from the total intersection cycles. Since $W^{(d-1)}$ is irreducible, our $|E|$ has no fixed cycle, unless $r=0$ in which case $|E|$ consists of $W^{(d-1)}$ alone. (This happens for example when $W^{(1)}$ is one of the 27 straight lines on a cubic surface without singularities.) We make the special assumption that there does not exist a $(d-2)$-dimensional subvariety of $V^{(d)}$ which belongs to all cycles of $|E|$; this clearly is stronger than assuming that $r>0$. Under this assumption, the linear series $g^{r-1}$, cut out on $E$ by the cycles of $|E|$, can be defined as follows, avoiding all questions of intersection theory we have not dealt with already. Among the above forms $k_{0}(x), \cdots, k_{r}(x)$ there are precisely $r$ forms, say $k_{0}(x), \cdots$, $k_{r-1}(x)$, which are linearly independent modulo $W^{(d-1)}$. The $g^{r-1}$ cut out on $E$ by $|E|$ is exactly the linear series without fixed cycle cut out on $W^{(d-1)}$ by the hypersurfaces $\lambda_{0} k_{0}(x)+\cdots+\lambda_{r-1} k_{r-1}(x)=0$. (This may of course necessitate subtracting a fixed, effective, $(d-2)$-dimensional cycle of $W^{(d-1)}$ from the total intersection divisors of $W^{(d-1)}$ with the hypersurfaces $\lambda_{0} k_{0}(x)+\cdots+\lambda_{r-1} k_{r-1}(x)=0$.) The fact that $|E|$ is complete does not imply that $g^{r-1}$ is complete; on the contrary, the deficiency of $g^{r-1}$ may very well be positive.

EXAMPLES 6.1. $V^{(3)}$ is all of 3 -space and $W^{(2)}$ is one of its planes. $|E|$ now consists of all planes of 3 -space and hence $r=3$. The $g^{2}$ consists of all straight lines of $W^{(2)}$ and hence is complete. Now let $V^{(2)}$ be all of 2-space and $W^{(1)}$ an irreducible, third degree curve in $V^{(2)}$ with a double point. $|E|$ consists of all third degree curves of $V^{(2)}$ and hence $r=9$. The $g^{8}$ clearly has degree 9 ; that is, it is a $g_{9}^{8}$. Since our $W^{(1)}$ is a rational curve, this $g_{9}^{8}$ is not complete, but has deficiency $\delta=1$. Observe that, if we had chosen for our $W^{(1)}$ an irreducible third degree curve without singularities, the corresponding $g_{9}^{8}$ would have been complete.

REMARK. Whenever $W^{(s)}$ is an irreducible, $s$-dimensional subvariety of $V^{(d)}$ and $g$ a linear series of $V^{(d)}$, such that there does not exist an $(s-1)$-dimensional subvariety of $W^{(s)}$ which belongs to all 
cycles of $g$, the linear series of $W^{(s)}$ which results from intersecting $W^{(s)}$ with the cyles of $g$ can be defined as above.

Let us now return to the case $d=2$. We assume again that $V^{(2)}$ is an irreducible surface of 3-space with only ordinary singularities whose complex $K^{(4)}$ is homogeneous. We shall call an irreducible curve $W^{(1)}=E$ of $V^{(2)}$ "general" if:

(1) There exists on $V^{(2)}$ no point which belongs to all cycles of $|E|$.

(2) A tangent plane of $V^{(2)}$ contains at most one simple tangent of $W^{(1)}$.

The first condition guarantees that the linear series $g_{t}^{r-1}$ of $W^{(1)}$, obtained by intersecting $W^{(1)}$ with the cycles of $|E|$, can be defined as above. This $g_{t}^{r-1}$ is called the characteristic series of $W^{(1)}$ and its degree $t$ the virtual degree of $W^{(1)}$. The second condition states that on every two-dimensional branch of $V^{(2)}$ our curve $W^{(1)}$ behaves as a nonsingular curve. $V^{(2)}$ has general curves galore. For example, the intersection of $V^{(2)}$ with a hypersurface will almost always be a general curve. We are using here the term "general" as is customary in algebraic geometry. Precisely, if $\partial$ is large enough, all one-dimensional cycles of degree $\partial$ are general curves, except for perhaps those which correspond to the points of a subvariety $\Omega$ of the variety $\Pi$ which parametrizes all one-dimensional cycles of degree $\partial$; the important thing is that the dimension of $\Pi$ exceeds the dimension of $\Omega$. (The dimension of a reducible variety is defined as the largest dimension which occurs among its irreducible components.)

Castelnuovo has proved in [6] that the deficiencies of the characteristic series of the general curves of $V^{(2)}$ reach a finite maximum on $V^{(2)}$. This maximum is usually denoted by $q$ and is called the irregularity of the surface; when $q=0$, the surface is called regular, otherwise irregular. The Betti number $b_{1}=b_{3}$ of $K^{(4)}$ is equal to $2 q$ (see [3]), and hence we have now found an algebro-geometric definition for this Betti number. We see that the connected, orientable, closed, homogeneous, four-dimensional complexes, which arise from algebraic surfaces, are not the most general complexes of this type; a restriction on their topology is that $b_{1}$ is even. Since $q$ is the maximum number of simple integrals of the first kind of $V^{(2)}$ which are linearly independent modulo the constants, $q$ is a birational invariant of $V^{(2)}$. Observe from examples 6.1 that the second condition in the definition of a general curve can not be left out, when quoting the Castelnuovo theorem; namely, for the plane, $q=0 \mathrm{but}$, for the cubic curve with double point, the deficiency of its characteristic series is 1 . 
The following birational invariants also belong in the theory of linear equivalence. The singular points of $V^{(2)}$ fill up an algebraic curve. If $D_{1}^{(1)}, \cdots, D_{h}^{(1)}$ are the irreducible components of this curve, we denote by $D$ the one-dimensional cycle $D_{1}^{(1)}+\cdots+D_{h}^{(1)}$; if $V^{(2)}$ has no singularities, we consider $D$ as the zero cycle. The surfaces of 3-space which contain $D$, that is, which contain $D_{1}^{(1)}, \ldots, D_{h}^{(1)}$, are called the adjoints of $V^{(2)}$. If $m$ is the degree of $V^{(2)}$, the maximum number of linearly independent adjoints of degree $m-4$ is a birational invariant of $V^{(2)}$, called the geometric genus $p_{0}$ of $V^{(2)}$. Hence, the adjoints of degree $m-4$, if there are any, cut out on $V^{(2)}$ a linear series of dimension $p_{\theta}-1$ which contains at least $D$ as a fixed cycle; the $\left(p_{0}-1\right)$-dimensional linear series which is obtained by subtracting $D$ from all the total intersection cycles is called the impure canonical system $K$ of $V^{(2)}$. Every linear series of $V^{(2)}$, obtained by intersecting $V^{(2)}$ with all its adjoints of a fixed degree and subtracting $D$ from the total intersection cycles, is a complete linear series; in particular, $K$ is complete. The birational invariance of $p_{0}$ follows from the fact that the maximum number of double integrals of the first kind of $V^{(2)}$, which are linearly independent modulo the constants, is exactly $p_{g}$. The birational invariant $p_{0}-q$ is called the arithmetic genus $p_{a}$ of $V^{(2)} ; p_{a}$ may very well be negative.

It is usually not difficult to compute $p_{\theta}$ directly from its definition. We now show how $p_{a}$, and hence $q$, can be computed. The planes of 3 -space constitute an irreducible algebraic system of dimension 3 . Except for at most a subvariety of planes (that is, an algebraic system of planes) of dimension 2 , a plane intersects $V^{(2)}$ in an irreducible curve. The genus of such an irreducible plane curve of degree $m$ is of course at most $(m-1)(m-2) / 2$. We denote by $p$ the maximum genus of the irreducible plane curves of $V^{(2)}$ and by $\alpha$ the maximum number of linearly independent adjoints of degree $m-3$ of $V^{(2)}$. It follows from a theorem of Picard (see [7, vol. 2, p. 437]) that $p_{a}=\alpha-p$.

EXAMPLES 6.2. Let $V^{(2)}$ be a plane. Then $m=1$ and hence there exist no adjoints of degree $m-4$ or $m-3$; hence $p_{o}=\alpha=0$. Intersection of $V^{(2)}$ with a plane gives rise to a straight line; hence $p=0$, which shows that $p_{a}=q=0$. Hence, the three birational invariants $q, p_{g}, p_{a}$ are also zero for all rational surfaces. An example of a nonrational surface with $q=p_{0}=p_{a}=0$ is furnished by the sixth degree surfaces of Enriques. (See examples 4.1.) In that case, $m-4=2$; and, since there exist no quadrics which contain all six edges of a tetrahedron, $p_{g}=0$. A general plane intersects $V^{(2)}$ in a 6 th degree curve with 6 ordinary double points; hence, $p=4$. The four faces of the 
tetrahedron, taken three at a time, furnish four linearly independent adjoints of degree 3 , from which it follows that $\alpha=4$ and hence that $p_{a}=q=0$. All surfaces $V^{(2)}$ without singularities are regular. Thus, the number of linearly independent surfaces of degree $m-4$ is $(m-1)(m-2)(m-3) / 6=p_{g} ;$ in the same way, $\alpha=1 m(m-1)(m-2) / 6$. A general plane intersects $V^{(2)}$ in a nonsingular curve of degree $m$; hence $p=(m-1)(m-2) / 2$. It follows that $p_{a}=\alpha-p=(m-1)(m-2)$ $\cdot(m-3) / 6$ and hence that $q=0$. Ruled surfaces never have adjoints of degree $m-4$ or $m-3$, while $p$ can be arbitrarily large. Hence, these surfaces furnish examples of irregular surfaces, as now $p_{g}=0$, $p_{a}=-p, q=p$.

The only topological invariant of $K^{(4)}$ for which we have not yet given an algebro-geometric definition is the second Betti number $b_{2}$. We have seen that the straight lines of 3-space form a 4-dimensional irreducible algebraic system. The number of tangent planes of $V^{(2)}$ which pass through a straight line of 3-space is always the same, except for at most a 3-dimensional algebraic system of lines. This number is called the class $c$ of $V^{(2)}$. The Euler characteristic $b_{0}-b_{1}$ $+b_{2}-b_{3}+b_{4}$ of $K^{(4)}$ turns out to be $c-4 p-m+4$, where $p$ has the same meaning as above; this follows from a formula of Alexander (see Rendiconti dei Lincei, August 1914, and [5, p. 96]). Hence, $2-4 q+b_{2}=c-4 p-m+4$, which shows that $b_{2}=c-4 p-m+4 q+2$. This formula can be considered as an algebro-geometric definition of $b_{2}$ and enables us to compute $b_{2}$ easily.

EXAMPLES 6.3. If $V^{(2)}$ is a plane, $c=p=q=0$ and $m=1$; hence, $b_{2}=1$. Consequently, $\rho_{0}=0$ and since $\rho_{0}$ is a birational invariant (see $\S 4), \rho_{0}=0$ for all rational surfaces. We conclude for example that for the nonsingular quadrics, $b_{2}=2$.

We have not attempted to define all known birational invariants of surfaces. Equivalence relations have further usage, besides discovering and studying birational invariants. For example, the Riemann-Roch theorem for surfaces belongs properly in the theory of linear equivalence. Let $W^{(1)}$ be a general curve of genus $p$, and with characteristic series $g_{t}^{r-1}$. Let $i$ be the number of linearly independent cycles of the impure canonical system $K$ which contain $W^{(1)}$ as a component. Then the Riemann-Roch theorem asserts that $r \geqq t-p+p_{a}-i+1$.

7. Rational equivalence. It is true that, using only the part of intersection theory which is based on the notion of length of a primary ideal, we have been able to define several of the invariants of algebraic surfaces and state some of their properties. This should not mislead the reader into thinking that the algebrogeometric theory of 
surfaces can be developed without some thorough, general intersection theory. It is inconceivable that the assertions of the previous sections can be proved, using only intersection multiplicities which are based on the notion of length.

Let us return to our irreducible variety $V^{(d)}$. If $E_{1}, \cdots, E_{h}$ are $h$ effective, $(d-1)$-dimensional cycles of $V^{(d)}$ where $1 \leqq h \leqq d$, it is part of the task of intersection theory to define the $(d-h)$-dimensional intersection cycle $E_{1} \cdots \cdots E_{h}$ of $E_{1}, \cdots, E_{h}$. If $W_{1}^{(d-h)}, \cdots, W_{k}^{(d-h)}$ are the irreducible, $(d-h)$-dimensional components of $E_{1} \cdots E_{h}$ and $n_{1}, \cdots, n_{k}$ their respective intersection multiplicities, the cycle $E_{1} \cdots E_{h}$ is exactly $n_{1} W_{1}^{(d-h)}+\cdots+n_{k} W_{k}^{(d-h)}$. It is usually intuitively evident what $W_{1}^{(d-h)}, \cdots, W_{k}^{(d-h)}, n_{1}, \cdots, n_{k}$ are, but intersection theory tries to give definitions for them which are acceptable both from the point of view of mathematical rigor and mathematical simplicity. Let us suppose that this has been done, that is, that the intersection cycle $E_{1} \cdots \cdots E_{h}$ has been well defined.

Consider $h$ linear series $L_{1}, \cdots, L_{h}$ of $V^{(d)}, 1 \leqq h \leqq d$. Each cycle $E_{i}$ of $L_{i}$ corresponds to a point of the parametrizing projective space $\Omega_{i}$ of $L_{i}$, and hence to each point of the product space $\Omega_{1} \times \cdots \times \Omega_{h}$ we can associate the intersection cycle $E_{1} \ldots \ldots E_{h}$. The effective, $(d-h)$-dimensional cycles, obtained in this way, form an irreducible algebraic system, parametrized by $\Omega_{1} \times \cdots \times \Omega_{h}$ and called an elementary system of rational equivalence. When $h=1$, we obtain again the linear series and, as in the case of linear series, we allow subtraction of fixed cycles if they occur. For two effective, $(d-h)$-dimensional cycles $E_{1}, E_{2}$ we define that $E_{1} \sim E_{2}$ if they belong to the same elementary system of rational equivalence. The binary relation $\sim$ is reflexive and symmetric, but may not be additive. If it is not, we first go over to an additive relation $\approx$ for the effective, $(d-h)$ dimensional cycles by the procedure described in $\$ 2$; the covering $U$ now has as elements the elementary systems of rational equivalence. Then, by our standard method, we introduce an equivalence relation for the group $C_{d-h}$ of $(d-h)$-dimensional cycles which gives rise to a homomorphic image of $C_{d-h}$. The relation for the cycles of $C_{d-h}$, obtained in this way, is called rational equivalence. Hence, two arbitrary cycles $A, B \in C_{d-h}$ are rationally equivalent if there exists an $X \in C_{d-h}$ such that $A+X=E_{11}+\cdots+E_{1 k}, B+X=E_{21}+E_{22}$ $+\cdots+E_{2 k}$, where the effective cycles $E_{1 i} \sim E_{2 i}$ for $i=1, \cdots, k$. Rationally equivalent cycles clearly have the same degree and are necessarily algebraically equivalent; if $h=1$, rational equivalence coincides with linear equivalence.

The rational equivalence of pointgroups, that is, of 0 -dimensional 
cycles, is used in the proofs of the theorems we stated for surfaces. Efforts to extend these theorems to varieties of arbitrary dimension lead to outstanding problems of algebraic geometry and in this work the rational equivalence for arbitrary $C_{d-h}$ is used.

8. How to get started in algebraic geometry. This section is meant for readers who want to learn algebraic geometry but have to do this studying by themselves.

For most people, the easiest way to get into this field is probably through the so-called algebro-geometric method. This is the method whose main instruments come from projective geometry and modern algebra and which uses analysis and topology only as secondary tools; it is the method used in this exposition.

First, then, some knowledge of projective geometry and modern algebra has to be acquired. When selecting one of the many books on projective geometry, choose one which treats this subject from the technical rather than from the axiomatic point of view. A book of about 150 to 200 pages which defines $n$-dimensional projective space in terms of $(n+1)$-tuples, discusses general properties of $n$-dimensional space rather than special properties of the straight line, the plane, and 3-space, uses projective coordinates whenever they are convenient, and so on, is the sort of book best suited for the present purpose. As far as modern algebra is concerned, some knowledge of field extensions, valuation theory, and commutative ring theory should be obtained. It is not necessary to study any of these subjects extensively to get started in algebraic geometry. For example, $\$ \$ 6$ through 41,50 through 52 , and chapters $8,10,11,12,13$ of the English edition of van der Waerden's Modern algebra should be more than enough.

As a first book on algebraic geometry, try Algebraic geometry by S. Lefschetz which is announced as No. 18 of the Princeton Mathematical Series, to be published in June, 1953. This book makes tough, but rewarding, reading and is particularly strong in giving the reader an insight into the transcendental methods of curve and surface theory, although only the algebro-geometric method is used. When the reading gets too tough, the very clearly written Algebraic curves by R. J. Walker may be of help; this book is more elementary than the first and treats many of its subjects. Next, it is advisable to study volume 2 of Methods of algebraic geometry by W. V. D. Hodge and D. Pedoe. Here we find a good treatment of algebraic systems, algebraic equivalence, intersection theory, and so on. After these two books have been studied, the student ought to be willing to relax his mathematical rigor somewhat, so that he can get an insight into 
the over-all accomplishments of classical algebraic geometry and acquaint himself with the outstanding problems of present day algebraic geometry. There is no doubt that as yet this can not be done if we insist that every statement be proved with complete mathematical rigor, as we understand that term today. Excellent books for this purpose are reference [5] of this exposition and further literature given there.

Once the above material has been absorbed, the student should be ready to specialize in whatever direction he chooses. Besides the algebro-geometric direction, there are the algebraic direction and the transcendental direction.

If the student wants to go on in the algebro-geometric direction, he should read the papers by $\mathrm{O}$. Zariski in order to get an insight into how valuation theory is used in the study of varieties of arbitrary dimension.

The algebraic direction is the one where as little projective geometry as possible is used, where the groundfield is made as arbitrary as possible, and where one tries to use only algebra as his tool. Although the distinction between the algebro-geometric method and the algebraic method is necessarily vague, it is safe to say that the latter method is the one which is most important for algebraic number theory. The student who wants to pursue the strictly algebraic direction should begin by learning the abstract theory of algebraic function fields of one variable. An excellent account of this theory can be found in Algebraic numbers and algebraic functions by E. Artin; this is a set of lecture notes, mimeographed in 1950-1951 by New York University. The student should then study Foundations of algebraic geometry by A. Weil, published as vol. 29 of the American Mathematical Society Colloquium Publications.

The transcendental direction is the one which is based on analysis and topology. There one can get a start by studying in references [3] and [7] of this exposition. As a preparation for [7], the Traité d'Analyse by $\mathrm{E}$. Picard is helpful. Except for a 1934-1935 mimeographed set of Princeton lectures, given by S. Lefschetz and written by N. E. Steenrod and H. Wallman, the author is not able to mention an efficient preparation for [3].

\section{BIBLIOGRAPHY}

1. G. Mannoury, Surface-images, Nieuw Archief van Wiskunde (2) vol. 4 (1898) pp. 112-129.

2. W. L. Chow and B. L. van der Waerden, Zur algebraischen Geometrie. IX. Über zugeordnete Formen und algebraische Systeme von algebraischen Mannigfaltigkeiten, Math. Ann. vol. 113 (1937) pp. 692-704. 
3. S. Lefschetz, L'analysis situs et la géometrie algêbrique, Paris, 1924 and 1950.

4. B. L. van der Waerden, On Hilbert's function, series of composition of ideals and a generalization of the theorem of Bezout, Neder. Akad. Wetensch. vol. 31 (1928) pp. 749-770.

5. F. Severi, Fondamenti di geometria algebrica, Padova, 1948.

6. G. Castelnuovo, Alcuni risultati sui sistemi lineari di curve appartenenti ad una superficie algebrica, Memorie della Societa Italiana dell Scienze Detta Dei XL (3) vol. 10 (1896) pp. 82-102; Alcune proprietd fondamentali dei sistemi lineari di curve tracciate sopra una superficie algebrica, Annali di Mathematica Pura ed Applicata (2) vol. 25 (1897) pp. 235-318.

7. E. Picard and G. Simart, Théorie des fonctions algêbriques de deux variables indépendantes, 2 vols., Paris, 1897-1906.

\section{University of Southern California}

